\title{
ERCC6L promotes cell growth and invasion in human colorectal cancer
}

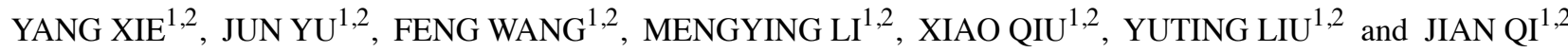 \\ ${ }^{1}$ Department of Gastroenterology, Zhongnan Hospital of Wuhan University; \\ ${ }^{2}$ Hubei Clinical Center and Key Laboratory of Intestinal and Colorectal Diseases, Zhongnan Hospital of \\ Wuhan University, Wuhan, Hubei 430071, P.R. China
}

Received March 19, 2018; Accepted April 4, 2019

DOI: $10.3892 / \mathrm{ol} .2019 .10297$

\begin{abstract}
Excision repair cross-complementation group 6 like (ERCC6L), a recently discovered DNA helicase, has been demonstrated to be highly expressed in a variety of human cancer types. However, the precise role of ERCC6L in colorectal cancer (CRC) remains unclear. The current study aimed to investigate the potential role of ERCC6L in the development and progression of CRC. Reverse transcription-quantitative polymerase chain reaction, western blot analysis and immunohistochemistry were used to detect the expression level of ERCC6L in 30 matched pairs of CRC and adjacent noncancerous tissues. The function of ERCC6L in cell proliferation, cycle, apoptosis, invasion and colony formation was examined in CRC cell lines. ERCC6L was revealed to be highly expressed in CRC tissues and cell lines compared with normal controls $(\mathrm{P}<0.05)$. The expression level of ERCC6L was significantly associated with tumor size $(\mathrm{P}<0.05)$, but not with other clinical features, including age, gender, differentiation and clinical stage. It was identified that reducing ERCC6L expression using small interfering RNA significantly inhibited the proliferation and colony-forming ability of CRC cell lines. Flow cytometric analysis demonstrated that ERCC6L knockdown in CRC cells inhibited cell cycle progression and increased the number of cells in the G0/G1 phase without affecting apoptosis. Furthermore, ERCC6L knockdown markedly decreased the number of invading CRC cells compared with control cells. These results suggest that ERCC6L promotes the growth and invasion of CRC cells, and ERCC6L may be a potential new target for cancer therapy.
\end{abstract}

Correspondence to: Professor Jian Qi, Department of Gastroenterology, Zhongnan Hospital of Wuhan University, 169 Donghu Road, Wuchang, Wuhan, Hubei 430071, P.R. China E-mail: qijian@whu.edu.cn

Key words: excision repair cross-complementation group 6 like, colorectal cancer, immunohistochemistry, RNA interference, proliferations

\section{Introduction}

Tumors are the second leading cause of mortality worldwide, behind cardiovascular disease $(1,2)$. In the USA, the incidence and mortality rates of colorectal cancer (CRC) are the third highest among all cancer types in both males and females in 2018 (2). In China, CRC ranks as the fifth most common malignancy in males and the fourth most common malignancy in females, with 12,000 new cases being diagnosed each year (3). Early prevention, detection and treatment are crucial for cancer control and management. Although significant progress has been made in understanding the molecular mechanisms involved in CRC, and in CRC diagnosis and treatment, the 5-year survival rate of patients with CRC has not significantly improved (4-6). Therefore, the early detection of $\mathrm{CRC}$ for prognostic management with non-invasive methods is urgently necessary.

The nucleotide excision repair (NER) mechanism serves an important role in maintaining the integrity of chromosomes. Any factor that interferes with the NER mechanism can affect cellular activity and lead to cell death. The normal function of NER factors is particularly important for cell division and differentiation (7). The SNF2 family is an important family of proteins that function as NER factors and are essential for ribosome assembly, translation initiation and cell growth (7). Excision repair cross-complementation group 6 (ERCC6) is a member of the SNF2 family and serves a key role in transcription-coupled DNA repair, allowing access of the DNA-repair apparatus to DNA (7). ERCC6 is upregulated in CRC tissues, and increased expression levels of ERCC6 have been associated with a poor response to chemotherapy and worse survival for patients with CRC (8). A newly identified ERCC6-like gene in mice, ERCC6L, also known as polo-like kinase 1 (PLK1)-interacting checkpoint helicase, has been demonstrated to be a development-associated member of the SNF2 family $(9,10)$. Nielsen et al (9) identified that ERCC6L cooperates with topoisomerase II in mitosis to promote sister chromatid disjunction. Furthermore, ERCC6L has been revealed to function as a DNA-dependent ATPase that interacts with PLK to form a complex that maintains the chromosome architecture during prometaphase (10). These studies suggest that ERCC6L is not a typical NER factor but rather serves a role in the segregation of sister chromatids during mitosis. 
The ERCC6L protein is assembled in the cytoplasm and then enters the cell nucleus to exert its function (10). It is strongly expressed in the mouse embryonic stage, particularly in embryonic brain, heart, kidney, liver and lung. However, ERCC6L expression is significantly downregulated following birth, with no expression detected in the majority of adult organs $(7,11)$. Previous studies have demonstrated that ERCC6L is highly expressed in numerous types of human solid tumor; therefore, it is considered a potential target for cancer therapy $(12,13)$. $\mathrm{Pu}$ et al $(13)$ demonstrated that the mRNA expression level of ERCC6L increases during the progression of breast and kidney cancers, and increased ERCC6L expression is associated with poor overall survival. In addition, ERCC6L silencing has been revealed to inhibit the proliferation of cancer cells, which suggests that ERCC6L may be an effective biomarker for cancer progression (13). However, to the best of our knowledge, the role of ERCC6L in CRC remains to be investigated.

The present study first investigated the expression of ERCC6L in CRC tissues using immunohistochemistry (IHC), reverse transcription-quantitative polymerase chain reaction (RT-qPCR) and western blot analysis with the aim of determining the role of ERCC6L in CRC. Subsequently, the associations between ERCC6L expression level and the clinicopathological characteristics of patients with CRC were evaluated. Finally, the functional role of ERCC6L in CRC cell proliferation, cycle, apoptosis and invasion was explored by in vitro experiments.

\section{Materials and methods}

Tissue specimens. The present study included 30 patients with primary CRC from the Department of Colorectal Surgery at the Zhongnan Hospital of Wuhan University (Wuhan, China) from June to Setember 2017. Written informed consent was obtained from all patients prior to enrolment in the study. This prospective study was approved by the institutional Ethics Committee of Zhongnan Hospital of Wuhan University (Wuhan, China) and conducted in accordance with the ethical guidelines of the Declaration of Helsinki. All patients underwent surgical resection without preoperative chemotherapy and radiotherapy. Detailed patient clinical information is listed in Table I. The cohort consisted of 11 females and 19 males, with a median age of 51 years (range, 22-80 years). The diagnosis of all patients was confirmed to be CRC by histopathology. The tissue size as $1 \mathrm{~cm}^{3}$ and was stored in a liquid nitrogen until subsequent use.

Cell cultures. Human CRC (HCT116, SW480 and HT29) and normal colonic mucosal (NCM460) cell lines were obtained from The Cell Bank of the Chinese Academy of Sciences (Shanghai, China). All cells were cultured in Dulbecco's modified Eagle's medium (DMEM; Gibco; Thermo Fisher Scientific, Inc., Waltham, MA, USA) with $10 \%$ fetal bovine serum (FBS; Hangzhou Sijiqing Biological Engineering Materials Co., Ltd., Hangzhou, China) and 1\% penicillin/streptomycin. Cells were incubated in a humidified chamber with $5 \% \mathrm{CO}_{2}$ at $37^{\circ} \mathrm{C}$.

RNA interference and transfection. Small interfering RNA (siRNA) sequences targeting human ERCC6L
(siRNA-ERCC6L) and a negative control (si-NC) were purchased from Guangzhou RiboBio Co., Ltd. (Guangzhou, China). The sequences of the siRNAs were as follows: si-ERCC6L-101 forward, 5'-GCAGGCTGCTCATTACCT A-3' and reverse, 5'-TAGGTAATGAGCAGCCTGC-3'; si-ERCC6L-102 forward, 5'-GTAGGTGGTGTCGGTTTA A-3' and reverse, 5'-TTAAACCGACACCACCTAC-3'; si-ERCC6L-103 forward, 5'-GCTGGTTAATGACGTCTA A-3' and reverse, 5'-TTAGACGTCATTAACCAGC-3'; and NC forward, 5'-UUCUCCGAACGUGUCACGUTT-3' and reverse, 5'-ACGUGACACGUUCGGAGAATT-3'. SW480 and HT29 cells were seeded in a 6-well plate at a density of 50,000 cells $/ \mathrm{ml}$ and transfection was initiated when the cell confluence reached $50-60 \%$. The amount of siRNA transfected was 5.0 pmoles. Cells were transfected with the siRNA using GenMute ${ }^{\mathrm{TM}}$ siRNA Transfection Reagent (SignaGen Laboratories, Rockville, MD, USA), according to the manufacturer's protocol. Following $\sim 24 \mathrm{~h}$, cells were harvested for further experiments.

$R N A$ isolation and $R T-q P C R$. CRC tissue $\left(0.5 \mathrm{~cm}^{3}\right)$ was placed in an Eppendorf tube containing $1 \mathrm{ml}$ TRIzol reagent (Thermo Fisher Scientific, Inc.) and then ground with a homogenizer. Total RNA extraction was performed according to the manufacturer's protocol. The resulting RNA was dissolved in RNase-free water and immediately stored at $-80^{\circ} \mathrm{C}$. The RNA concentration was measured using a NanoDrop 2000 spectrophotometer (Thermo Fisher Scientific, Inc.). Complementary DNA was then synthesized using a ReverTra Ace qPCR RT kit (Toyobo Life Science, Osaka, Japan).qPCR was then performed using SYBR-green PCR master mix (CWBIO, Beijing, China) in a Bio-Rad 7500 real-time PCR system (Applied Biosystems; Thermo Fisher Scientific, Inc.). The reverse transcription reaction was carried out at $37^{\circ} \mathrm{C}$ for $15 \mathrm{~min}$, then the enzyme inactivation at $98^{\circ} \mathrm{C}$ for $5 \mathrm{~min}$, and finally stored at $4^{\circ} \mathrm{C}$. The primers for ERCC6L and GAPDH were purchased from Qingke Biotechnology Co., Ltd. (Wuhan, China). The specific primers used were as follows: ERCC6L forward, 5'-ATCGGT GCCTCAGCGTTCGG-3' and reverse, 5'-CTGTCCTCGCCG TCACACCG-3'; and GAPDH forward, 5'-AGAAGGCTG GGGCTCATTTG-3' and reverse, 5'-GCAGGAGGCATT GCTGATGAT-3'. GAPDH was used as a control. The reaction conditions were as follows: Pre-denaturation at $95^{\circ} \mathrm{C}$ for $10 \mathrm{~min}$; denaturation at $95^{\circ} \mathrm{C}$ for $10 \mathrm{sec}$; annealing at $60^{\circ} \mathrm{C}$ for $20 \mathrm{sec}$; extension at $72^{\circ} \mathrm{C}$ for $10 \mathrm{sec}$; full extension at $72^{\circ} \mathrm{C}$ for $3 \mathrm{~min}$; the number of gene cycles was 39 . All reactions were run in triplicate, and the results were analyzed and expressed relative to threshold cycle $(\mathrm{Cq})$ values and then converted to fold change values $\left(2^{-\Delta \Delta \mathrm{Cq}}\right)(14)$.

Western blot analysis. CRC tissues and cells were lysed with radioimmunoprecipitation assay protein extraction reagent (Beyotime Institute of Biotechnology, Haimen, China) supplemented with $1 \%$ phenylmethanesulfonyl fluoride (Seebio Science \& Technology Co., Ltd., Shanghai, China). Protein concentrations were measured using an enhanced BCA protein assay kit (Beyotime Institute of Biotechnology). Each lane was loaded with an equal amount of protein $(30 \mu \mathrm{g})$, and proteins were then separated using $8 \%$ SDS-PAGE and transferred to a polyvinylidene fluoride membrane (EMD Millipore, Billerica, 
Table I. Association of ERCC6L expression with clinicopathological parameters.

\begin{tabular}{|c|c|c|c|c|}
\hline \multirow[b]{2}{*}{ Characteristics } & \multirow[b]{2}{*}{ Cases, $\mathrm{n}$} & \multicolumn{2}{|c|}{$\begin{array}{l}\text { ERCC6L } \\
\text { expression }\end{array}$} & \multirow[b]{2}{*}{ P-value } \\
\hline & & $\begin{array}{l}\text { Low } \\
(n=9)\end{array}$ & $\begin{array}{l}\text { High } \\
(\mathrm{n}=21)\end{array}$ & \\
\hline Age, years & & & & 0.589 \\
\hline$<51$ & 8 & 3 & 5 & \\
\hline$\geq 51$ & 22 & 6 & 16 & \\
\hline Sex & & & & 0.563 \\
\hline Male & 19 & 5 & 14 & \\
\hline Female & 11 & 4 & 7 & \\
\hline Median tumor size, $\mathrm{cm}$ & & & & 0.011 \\
\hline$<5$ & 10 & 6 & 4 & \\
\hline$\geq 5$ & 20 & 3 & 17 & \\
\hline Tumor differentiation & & & & 0.109 \\
\hline Well/moderately & 25 & 6 & 19 & \\
\hline Poor & 5 & 3 & 2 & \\
\hline TNM stage & & & & 0.523 \\
\hline I-II & 16 & 4 & 12 & \\
\hline III-IV & 14 & 5 & 9 & \\
\hline
\end{tabular}

ERCC6L, excision repair cross-complementation group 6 like.

MA, USA). The membranes were blocked with $5 \%$ milk for $2 \mathrm{~h}$ at room temperature. The membranes were then placed in a TBS and Tween-20 (TBST) solution containing anti-ERCC6L antibody (1:1,000; cat. no. BC008808; Wuhan Sanying Biotechnology, Wuhan, China) and were allowed to react at $4^{\circ} \mathrm{C}$ overnight for $\sim 12 \mathrm{~h}$. The following day, following washing with TBST, the blots were incubated with a horseradish peroxidase-labeled anti-rabbit secondary antibody (1:5,000; cat. no. GB233303-1; Servicebio, Woburn, MA, USA) for $2 \mathrm{~h}$ at room temperature. The blots were visualized using a Super ECL detection reagent (Beijing Solarbio Science \& Technology Co., Ltd., Beijing, China). Each set of analyses was repeated a minimum of three times.

Wound healing assay. CRC cells were seeded in a 6-well plate (50,000 cells $/ \mathrm{ml})$. At $\sim 24 \mathrm{~h}$ post-transfection, the cells had reached $70-80 \%$ confluency. A straight scratch was created using a sterile pipette tip. PBS was added to the six-well plate to remove floating cells. The original culture medium was replaced with $0.5 \%$ FBS for a further $24 \mathrm{~h}$. All tests were repeated three times. Cell migration was observed and imaged at 0 and $24 \mathrm{~h}$ with a light microscope (x40 magnification; OLYMPUS U-RFL-T; Olympus Corporation, Tokyo, Japan).

Cell proliferation assay. At $24 \mathrm{~h}$ post-transfection, HT29 and SW480 cells were trypsinized to provide suspensions that were then seeded in 96-well plates at densities of 3,000 and 2,000 cells/well, respectively. The cell proliferation rates were calculated using the MTT method at $0,24,48,72$ and $96 \mathrm{~h}$. Briefly, $10 \mu \mathrm{l}$ MTT was added to each well and the cells were incubated for $4 \mathrm{~h}$. After $4 \mathrm{~h}$, the medium was replaced with $200 \mu \mathrm{l}$ dimethyl sulfoxide to dissolve the formazan. The absorbance value of each well at $490 \mathrm{~nm}$ was recorded. Each experiment was repeated a minimum of three times.

Colony formation assay. The colony formation ability of SW480 and HT29 cells transfected with siRNA was measured as follows. Cells were plated in 6-well plates at 1,000 cells/well and maintained in DMEM containing 10\% FBS. The cells were incubated in a humidified chamber with $5 \% \mathrm{CO}_{2}$ at $37^{\circ} \mathrm{C}$. Following 12-14 days, the cells were washed twice with PBS, fixed with $4 \%$ paraformaldehyde for $25 \mathrm{~min}$ and then stained with $0.1 \%$ crystal violet for $20 \mathrm{~min}$ at room temperature. All experiments were performed in triplicate.

Cell invasion assay. A 24-multiwell insert plate with a small chamber (BD Biosciences, San Jose, CA, USA) containing an $8.0-\mu \mathrm{m}$ pore size Matrigel-coated membrane was used for the cell invasion assays. Briefly, $2 \times 10^{5}$ cells in serum-free medium were seeded into the upper chambers, which were coated in Matrigel. The lower chambers of the 24-well plates were filled with $600 \mu 1$ DMEM containing $20 \%$ FBS as a chemo-attractant. Following incubation of the plates at $37^{\circ} \mathrm{C}$ for $24 \mathrm{~h}$, the non-invasive cells above the chamber were gently wiped away with a wet cotton swab. The cells in the lower chamber were fixed with $4 \%$ paraformaldehyde for $30 \mathrm{~min}$, stained with $0.1 \%$ crystal violet for $20 \mathrm{~min}$ at room temperature and then counted under a light microscope (x 200 magnification). Each experiment was repeated a minimum of three times.

Analysis of cell apoptosis and the cell cycle. The cells were seeded in 6 -well plates at $5 \times 10^{5}$ cells/well. Following transfection with siRNA for $24 \mathrm{~h}$, HT29 and SW480 cells were harvested, washed with PBS, resuspended in binding buffer and incubated at room temperature for $15 \mathrm{~min}$ in the dark with propidium iodide (PI) and annexin V/fluorescein isothiocyanate (Absin Bioscience Inc., Shanghai, China). The apoptosis rate was immediately detected by flow cytometry using a flow cytometer (Beckman CytoFLEX FCM, USA).

For analysis of the cell cycle, HT29 and SW480 cells were transfected with siRNA for $24 \mathrm{~h}$ and then fixed in $70 \%$ ethanol at $-20^{\circ} \mathrm{C}$ for $24 \mathrm{~h}$. The cells were washed twice with PBS and then incubated with RNase A at $37^{\circ} \mathrm{C}$ for $30 \mathrm{~min}$. PI (Nanjing KeyGen Biotech Co., Ltd., Nanjing, China) was added to the cell suspension $(100,000 / \mathrm{ml})$ and the cells were incubated for $30 \mathrm{~min}$ at room temperature in the dark. The cell cycle distribution was then analyzed using a flow cytometer. All experiments were performed in triplicate.

IHC. CRC tissues were fixed in $10 \%$ neutral buffered formalin solution for $24 \mathrm{~h}$ at room temperature and then embedded in paraffin for IHC analysis. Briefly, 5- $\mu$ m paraffin sections were deparaffinized in xylene and gradually rehydrated in 100, 95 and $75 \%$ ethanol at $60^{\circ} \mathrm{C}$. The $5-\mu \mathrm{m}$ paraffin sections were baked in a $60^{\circ} \mathrm{C}$ oven for $2 \mathrm{~h}$, then deparaffinized in xylene and gradually rehydrated in 100, 95 and $75 \%$ ethanol. The tissue sections were blocked with $30 \% \mathrm{H}_{2} \mathrm{O}_{2}$ for 20 min at room temperature to quench the activity of endogenous peroxidases. Following antigen retrieval in heated $10 \mathrm{mM}$ citrate buffer for 
A

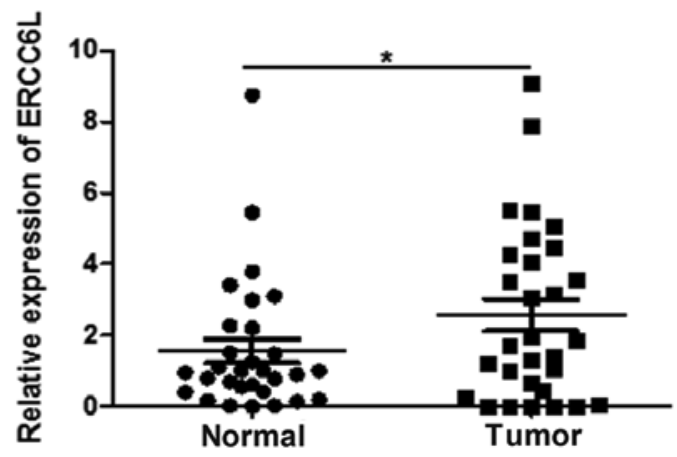

C

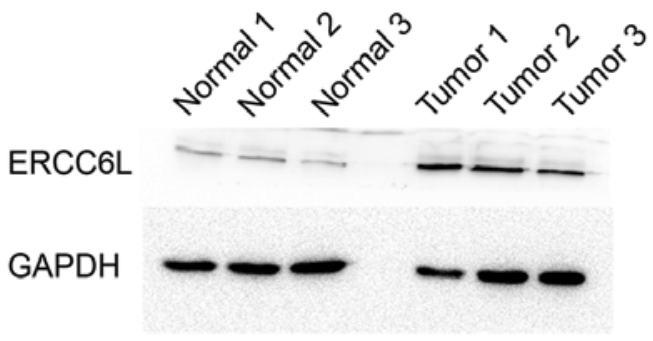

$\mathrm{B}$

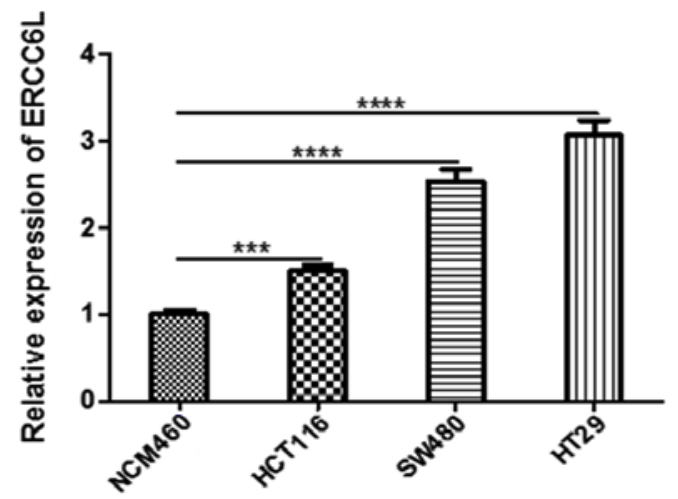

D

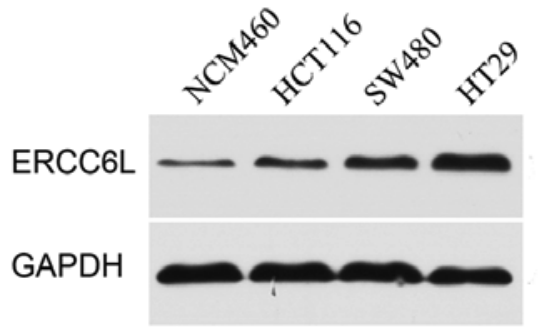

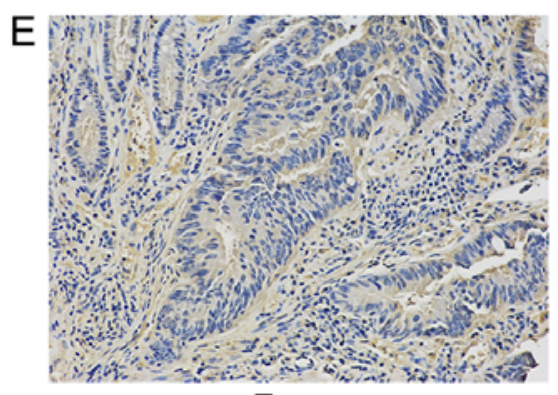

Tumor

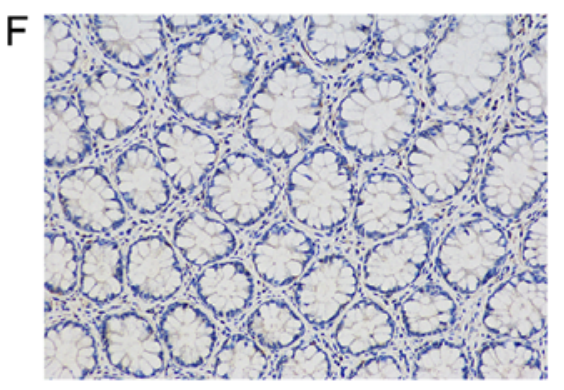

Normal

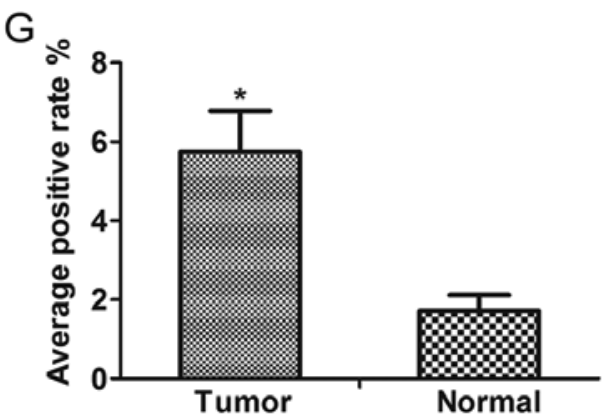

Figure 1. Analysis of ERCC6L expression in human CRC tissues and cell lines. (A) Reverse transcription-quantitative polymerase chain reaction was used to detect the relative expression levels of ERCC6L in 30 matched pairs of human CRC and noncancerous tissues. "P<0.05. (B) Relative expression of ERCC6L in three CRC cell lines (HCT116, SW480 and HT29) and one normal colonic mucosal cell line (NCM460). ${ }^{* * *} \mathrm{P}<0.001$ and ${ }^{* * * * *} \mathrm{P}<0.0001$. Expression level of ERCC6L in (C) three matched pairs of CRC and adjacent noncancerous tissues and (D) three CRC cell lines and one normal colonic mucosal cell line as detected by western blotting. GAPDH was used as an internal control. Representative images of IHC staining of ERCC6L in (E) a CRC tissue sample and (F) an adjacent normal tissue sample. Magnification, x200. (G) IHC revealed a significant upregulation of ERCC6L protein in tumor samples compared with normal samples. " $\mathrm{P}<0.05$ vs. normal. ERCC6L, excision repair cross-complementation group 6 like; CRC, colorectal cancer; IHC, immunohistochemistry.

$10 \mathrm{~min}$, the tissue sections were immunostained with primary antibody (anti-ERCC6L; 1:1,000; cat. no. BC008808) overnight at $4^{\circ} \mathrm{C}$. A mouse horseradish peroxidase-conjugated secondary antibody SignalStain ${ }^{\circledR}$ Boost IHC Detection Reagent (HRP, Rabbit) (1:1,000; cat no. 8114; Cell Signaling Technology, Inc.) was then added for $1 \mathrm{~h}$ at room temperature. Finally, images were observed under a light microscope (x 200 mangification). The positive cells were judged by the staining of nucleus and cytoplasm. The positive rate was divided by the total number of stained positive cells in different visual fields, and then the average value was taken for statistical analysis.

Statistical analysis. Data are presented as the mean standard \pm deviation. The IHC staining results and patient data were evaluated using a $\chi^{2}$ test. Other data presented in the text and figures were analyzed using one-way analysis of variance with a Bonferroni correction. All statistical analysis was performed using SPSS 22.0 (IBM Corp., Armonk, NY, USA).
$\mathrm{P}<0.05$ was considered to indicate a statistically significant difference.

\section{Results}

Expression of ERCC6L in CRC tissues and cell lines. The expression level of ERCC6L was detected in 30 paired samples of CRC and adjacent histological noncancerous tissues by RT-qPCR. As demonstrated in Fig. 1A, the expression level of ERCC6L in CRC tissues was significantly higher compared with the expression level in paracancerous tissues $(\mathrm{P}<0.05)$. Similarly, the expression levels of ERCC6L in a normal colonic mucosal (NCM460) and three CRC (HT29, SW480, HCT116) cell lines were determined by RT-qPCR. The expression level of ERCC6L in HT29, SW480 and HCT116 CRC cells was significantly higher compared with that in NCM460 cells ( $\mathrm{P}<0.001$; Fig. 1B). In addition, western blot analysis revealed that the protein expression 

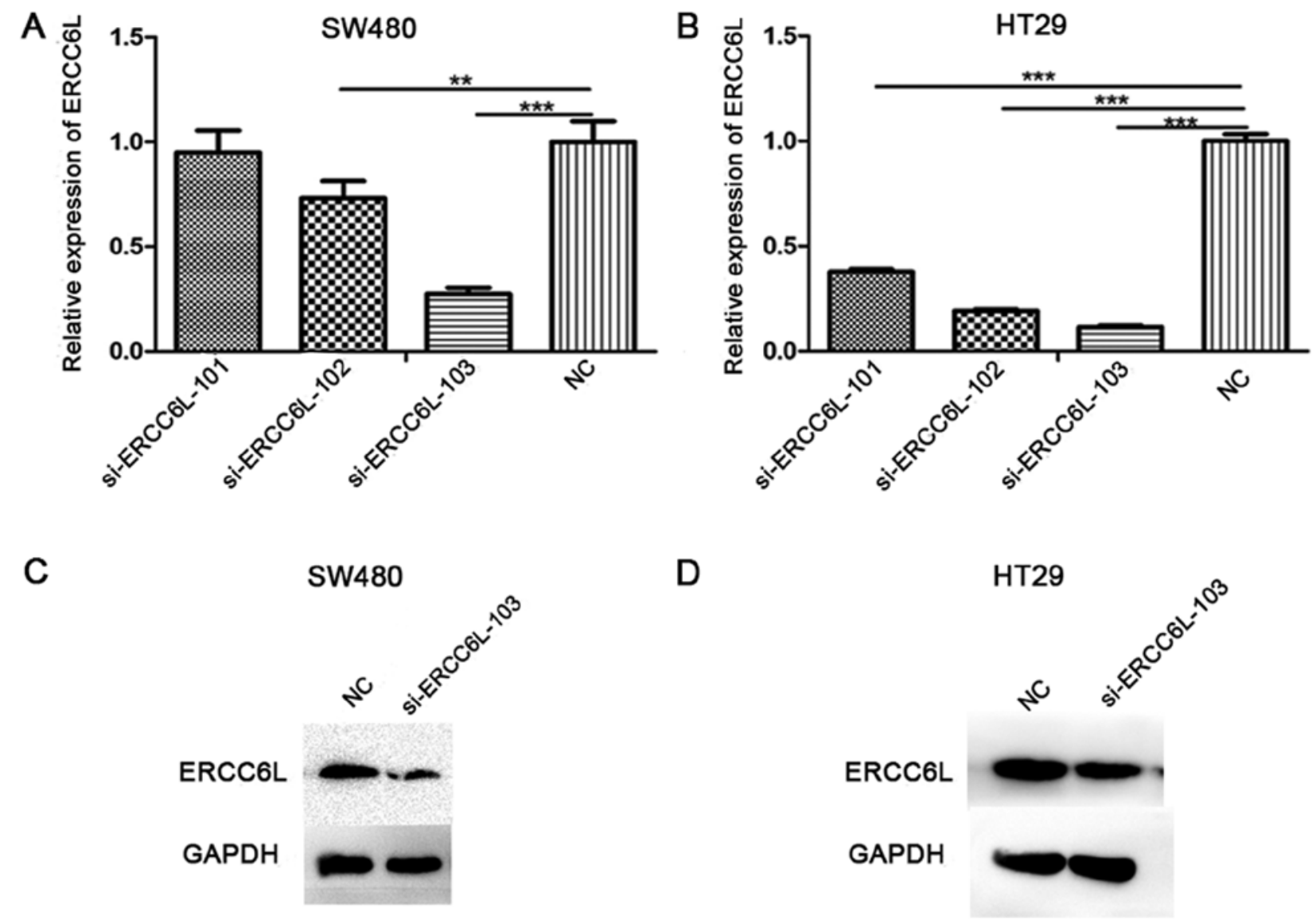

Figure 2. Silencing efficiency of three siRNA sequences targeting the ERCC6L gene as determined by RT-qPCR. GAPDH was used as an internal control. RT-qPCR analysis of mRNA in (A) SW480 cells and (B) HT29 cells transfected with ERCC6L siRNAs and NC siRNA. Western blot analysis of ERCC6L protein expression in (C) SW480 cells and (D) HT29 cells transfected with an ERCC6L siRNA and NC siRNA. GAPDH served as a loading control. "* P $<0.01$, ${ }_{* * * *} \mathrm{P}<0.001$. si, small interfering; NC, negative control; ERCC6L, excision repair cross-complementation group 6 like; RT-qPCR, reverse transcription-quantitative polymerase chain reaction.

level of ERCC6L was increased in CRC tissues and cancer cell lines compared with that in normal tissues and cells (Fig. 1C and D). IHC demonstrated that ERCC6L is expressed in the cytoplasm and nucleus of cells, and indicated that its cytoplasmic expression is higher than that of the nucleus (Fig. 1E and F). In addition, the IHC results revealed that the expression level of ERCC6L was higher in tumor tissues compared with the expression in matched non-tumor tissues $(\mathrm{P}<0.05$; Fig. 1G).

Association between ERCC6L expression and the clinicopathological features of patients with CRC. To examine the association between ERCC6L expression and the clinicopathological features of patients with CRC, the patients were divided into low $(n=9)$ and high $(n=21)$ ERCC6L expression groups based on comparison with the expression levels of ERCC6L in adjacent noncancerous tissues. High ERCC6L expression group, the expression of ERCC6L in tumor tissue was higher than that in paracancerous tissue; and low ERCC6L expression group, the expression of ERCC6L in tumor tissue was lower than that in paracancerous tissue. As presented in Table I, the expression level of ERCC6L exhibited a significant association with tumor size $(\mathrm{P}<0.05)$; however, no associations were detected with other clinical characteristics, including age, sex, tumor differentiation and clinical stage.
Migration, invasion and proliferation of CRC cells decreases following ERCC6L knockdown. ERCC6L expression was knocked down using three different siRNAs, and the silencing efficiency of these siRNAs was detected by RT-qPCR (Fig. 2A and B). The results demonstrated that si-ERCC6L-103 reduced the expression level of ERCC6L to the greatest extent in the SW480 and HT29 cell lines $(\mathrm{P}<0.001)$; therefore, this siRNA was selected for subsequent experiments. Similarly, at the protein level (Fig. 2C and D), si-ERCC6L-103 could significantly decrease the expression of ERCC6L in SW480 and HT29 cells.

Wound healing, Transwell, colony formation and proliferation assays were conducted to evaluate the effects of ERCC6L knockdown (Figs. 3 and 4). Wound healing assays revealed that knockdown of ERCC6L inhibited the migration of SW480 and HT29 cells compared with that in the respective control group $(\mathrm{P}<0.05$; Figs. $3 \mathrm{~A}$ and $\mathrm{B}, 4 \mathrm{~A}$ and $\mathrm{B})$. Transwell assays were performed to evaluate the effect of ERCC6L on the invasion capability of CRC cells. Knockdown of ERCC6L in SW480 and HT29 CRC cell lines significantly decreased the number of invading cells compared with the respective cells treated with $\mathrm{NC}(\mathrm{P}<0.001$; Figs. 3C, 4C and D). Clonogenic survival assays revealed a significant reduction in the colony formation efficiency of cells transfected with ERCC6L-siRNA compared with the control group $(\mathrm{P}<0.01$; Figs. 3D, $4 \mathrm{E}$ and $\mathrm{F})$. To detect the effect of ERCC6L knockdown on CRC cell proliferation, 
A

$\mathrm{Oh}$

$24 \mathrm{~h}$

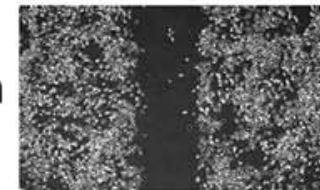

si-ERCC6L-103

C

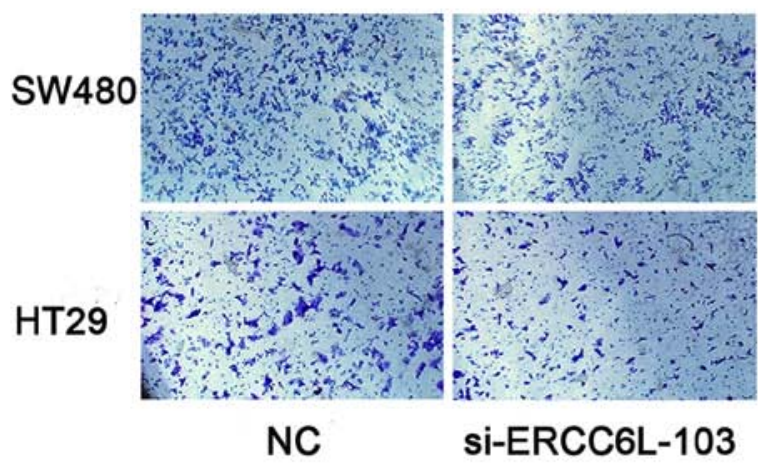

B

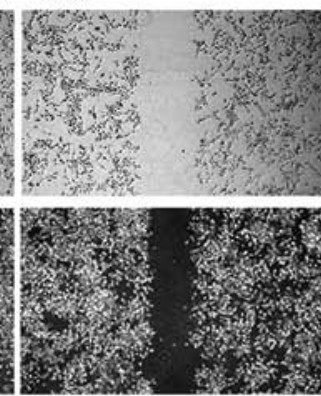

NC
$\mathrm{Oh}$

$24 \mathrm{~h}$

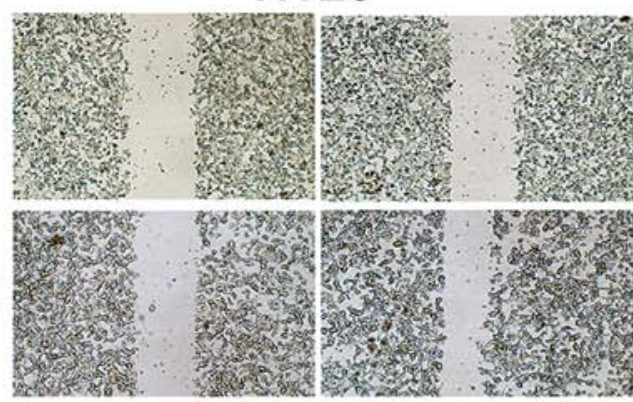

si-ERCC6L-103

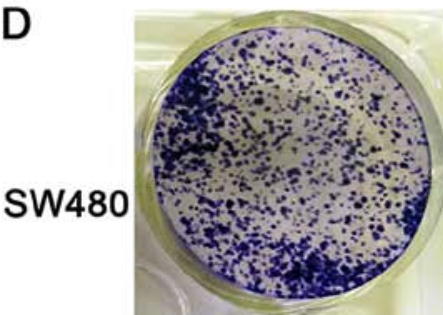

HT29

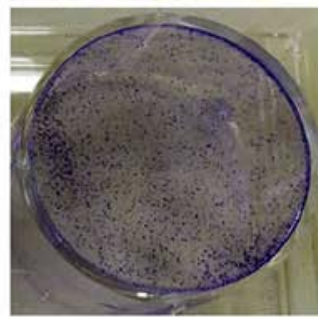

NC
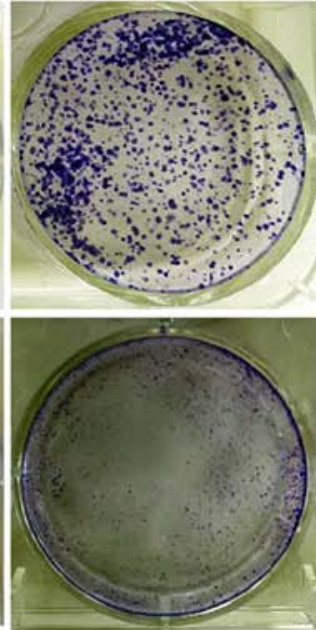

si-ERCC6L-103

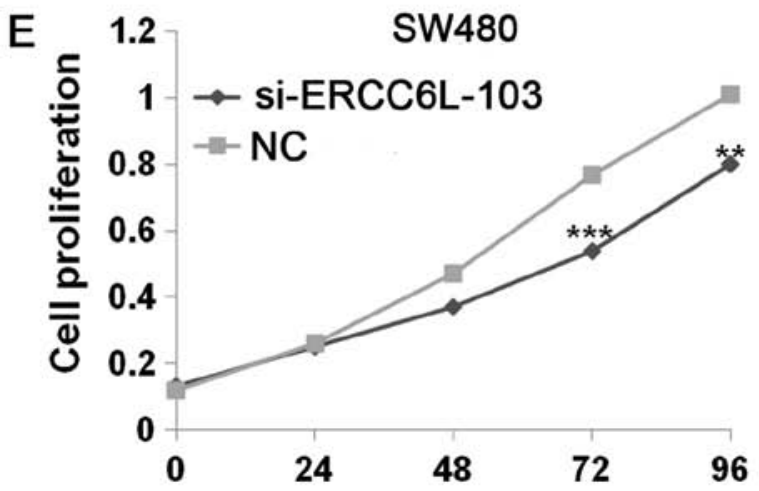

6 (h)

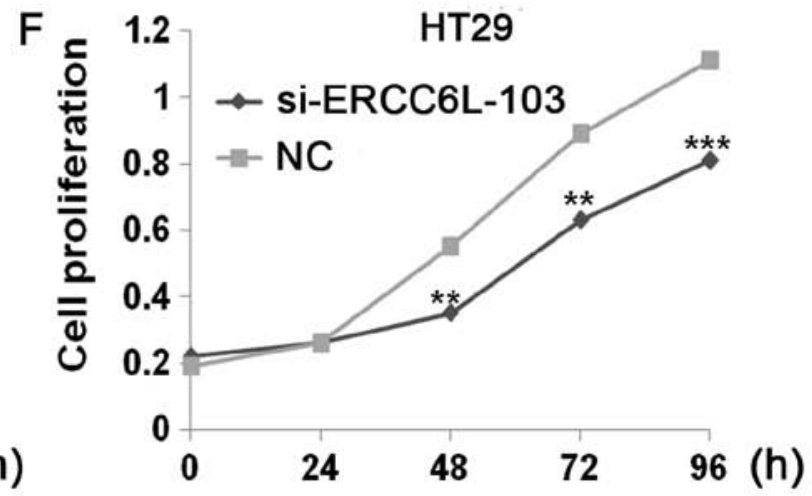

Figure 3. Effect of ERCC6L silencing on cell proliferation, migration and invasion. ERCC6L knockdown inhibited cell migration. Images of the same wounded area of transfected (A) SW480 cells and (B) HT29 cells 0 and $24 \mathrm{~h}$ after scratching (x40 magnification). (C) ERCC6L knockdown inhibited tumor cell invasion as demonstrated by a Transwell invasion assay. Magnification, x200. (D) Colony formation efficiency of transfected cells. Representative images of the plates used for colony enumeration. The effects of ERCC6L silencing on the proliferation of (E) SW480 and (F) HT29 cells as determined by MTT assay. ${ }^{* *} \mathrm{P}<0.01$,

${ }^{* * *} \mathrm{P}<0.001$ vs. NC. ERCC6L, excision repair cross-complementation group 6 like; NC, negative control; si, small interfering.

SW480 and HT29 cells were treated with ERCC6L-siRNA or a control siRNA for $72 \mathrm{~h}$, and cell viability was determined via an MTT assay. It was demonstrated that ERCC6L knockdown significantly inhibits CRC cell proliferation (Fig. 3E and F). These results indicate that ERCC6L promotes the migration, invasion and proliferation capability of CRC cells.

ERCC6L silencing induces cell cycle arrest at the G0/G1 phase in CRC cells. To evaluate the effect of ERCC6L on cell apoptosis, ERCC6L was knocked down in SW480 and
HT29 CRC cells, and apoptosis was then examined via flow cytometry (Fig. 5). The results revealed that cells treated with si-ERCC6L-103 and a siRNA control exhibited similar rates of apoptosis (Fig. 5A, B, E and F). Next, the effect of ERCC6L inhibition on the cell cycle in SW480 and HT29 cells was examined. The results demonstrated that ERCC6L silencing induced cell cycle arrest at the G0/G1 phase compared with the control group (Fig. 5C, D, G and H). In summary, these results indicate that ERCC6L silencing inhibits cell proliferation by blocking cells at the G0/G1 phase. 
A

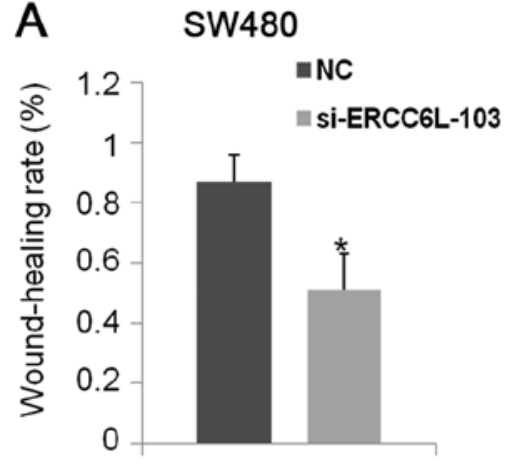

D

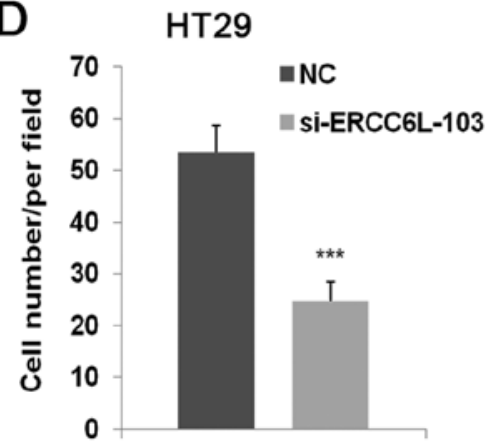

B

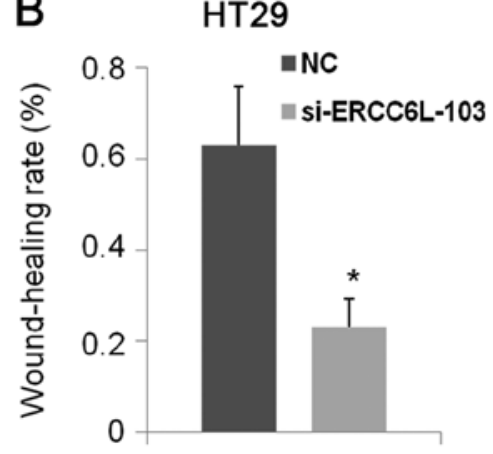

E

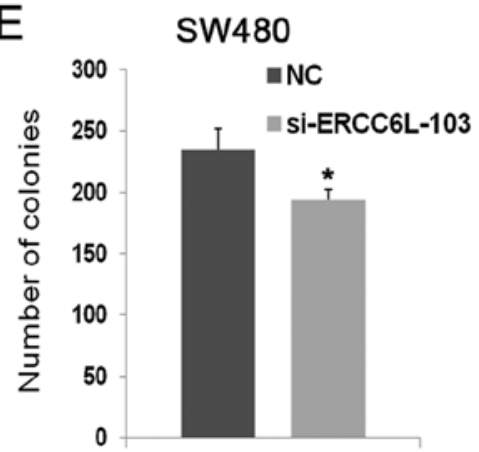

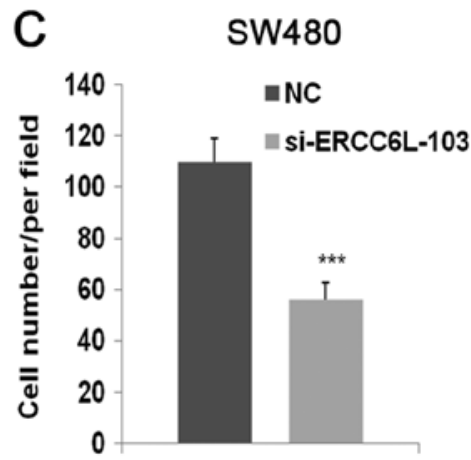

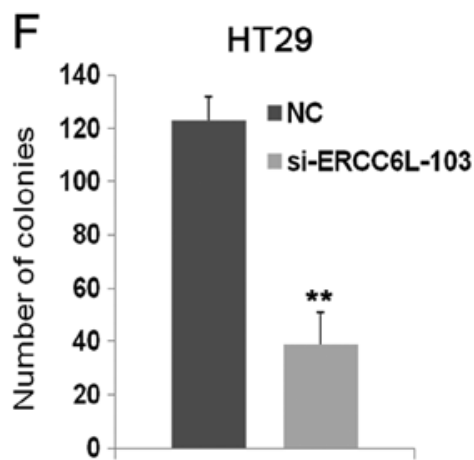

Figure 4. Quantification of the proliferation, migration and invasion assay results. The percentage of wound closure was calculated based on the cell migration distance relative to the initial wound distance for (A) SW480 and (B) HT29 cells. The number of invading (C) SW480 and (D) HT29 cells was calculated using an inverted light microscope. Analysis of the colony formation efficiency of (E) SW480 and (F) HT29 cells. ${ }^{*} \mathrm{P}<0.05,{ }^{* *} \mathrm{P}<0.01,{ }^{* * *} \mathrm{P}<0.001$ vs. NC. ERCC6L, excision repair cross-complementation group 6 like; NC, negative control; si, small interfering.

\section{Discussion}

CRC is a complex, multi-step disease with a variety of genetic alterations $(15,16)$. A number of proteins have been described as tumor biomarkers that aid the identification of CRC tumors, including A-kinase anchoring protein 4 (17), X-box binding protein 1 (18) and solute carrier family 38 member 1 (19). However, several different CRC subtypes have been identified, including subtypes based on DNA mismatch repair status, oncogene genotypes, or the consensus molecular subtypes derived from transcriptional profiling; therefore, one biomarker is unlikely to identify all types of colorectal tumor (20). Thus, it is essential to identify therapeutic targets and reliable prognostic biomarkers to improve the diagnosis and treatment of CRC.

ERCC6, a member of the SWI/SNF-associated ATPase family, has been associated with numerous diseases $(21,22)$. For example, it has been reported that Cockayne syndrome and cerebro-oculofacio-skeletal syndrome are caused by ERCC6 gene mutations (23-25). In addition, ERCC6 genetic polymorphism may provide early diagnosis, precise therapy, and improved clinical prognosis and quality of life in patients with bladder cancer (26). ERCC6L, another development-associated member of the SNF2 family, has a different function and role compared with ERCC6. A number of studies have examined the effects of disrupting ERCC6L function on chromosome structure and stability. For example, ERCC6L knockdown in human cancer cells was demonstrated to result in the loss of PLK1 from the chromosome arm and an increase in the occurrence of chromosomal abnormalities, including chromatin bridges and micronuclei (27-30). Furthermore, studies have revealed that PLK1 serves an important role in regulating biological processes, including apoptosis, the cell cycle and cell proliferation $(28,31-33)$. These findings indicate potential mechanisms by which ERCC6L regulates cellular biological functions.

The current study first used RT-qPCR and western blot analysis to detect the expression levels of ERCC6L mRNA and protein in CRC tissues and cell lines. In addition, IHC was used to detect the expression of ERCC6L protein in CRC tissues. The results revealed that the expression of ERCC6L was significantly increased in CRC compared with normal cells and tissues. Clinicopathological data confirmed that a high ERCC6L expression level was associated with increased tumor size. These findings suggest that ERCC6L overexpression may serve an important role in the development of CRC.

To investigate the role of ERCC6L in the development and progression of CRC, the expression of ERCC6L was inhibited using siRNA. It was identified that knockdown of ERCC6L led to the attenuated cell growth, cell cycle arrest, and decreased migration and invasion capability of CRC cells. However, the ability to migrate, invade and clone was higher in SW480 cells compared with HT29 cells, and a significant cell cycle arrest at G0/G1 was only observed in HT29 cells. These findings may result from differences in the KRAS and BRAF genotypes of these cell lines $(34,35)$. KRAS and BRAF have been demonstrated to be signal transduction molecules that serve a role in the mitogen-activated protein kinase pathway $(34,35)$. KRAS and BRAF gene mutations are understood to stimulate tumor growth and are present in various organs, including the 
A

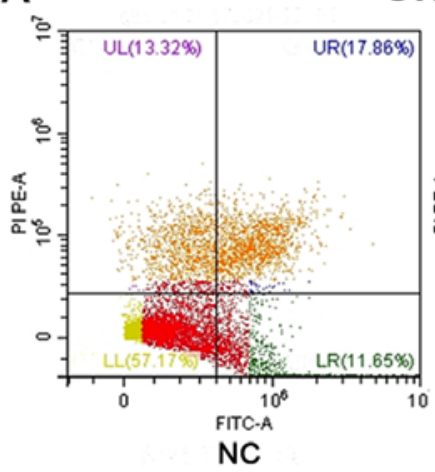

SW480

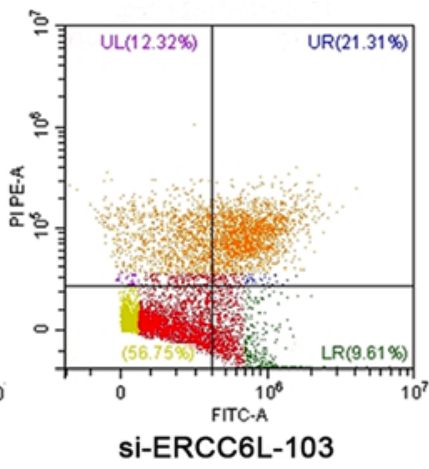

C

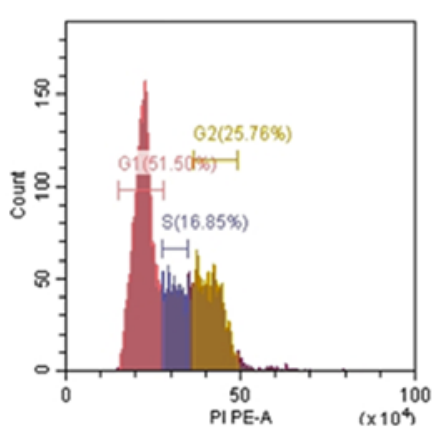

NC

$\mathrm{E}$

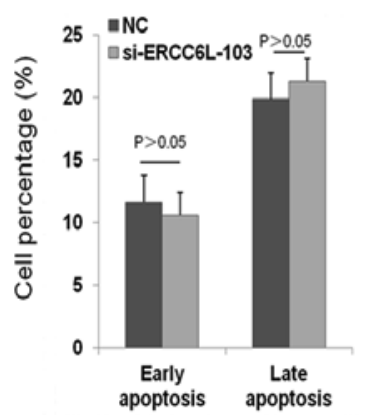

SW480

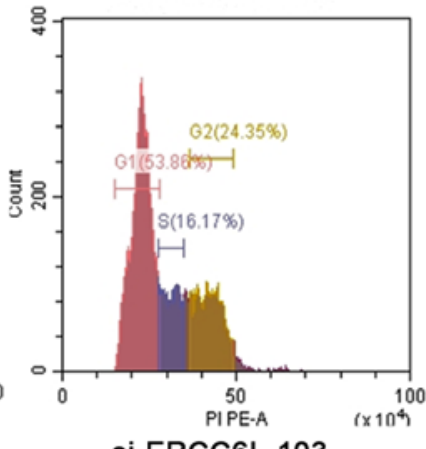

si-ERCC6L-103

$\mathrm{F}$

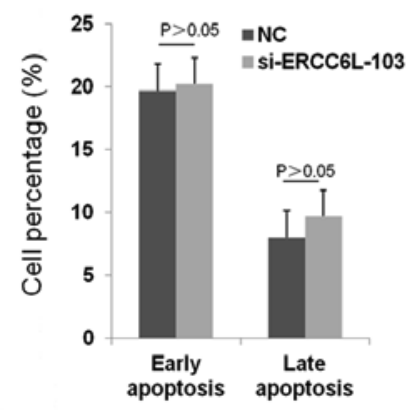

B

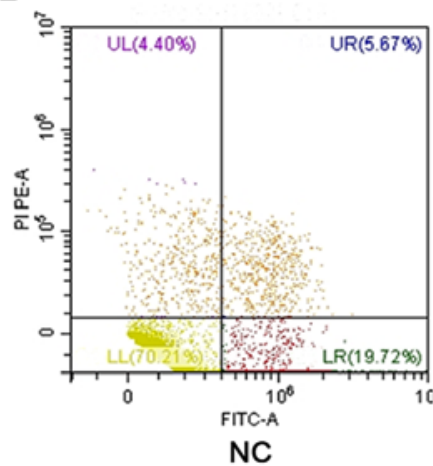

HT29

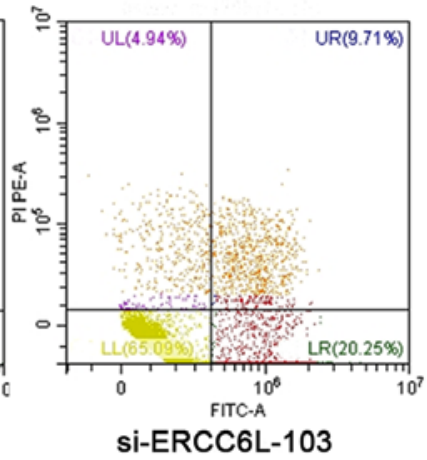

HT29

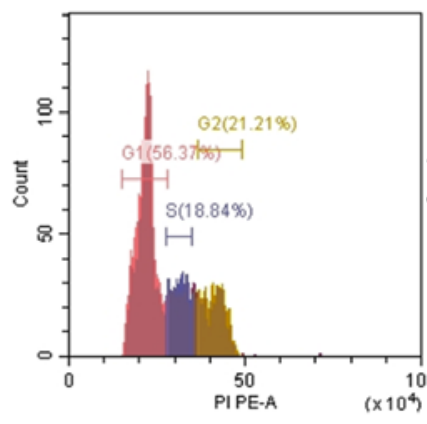

NC

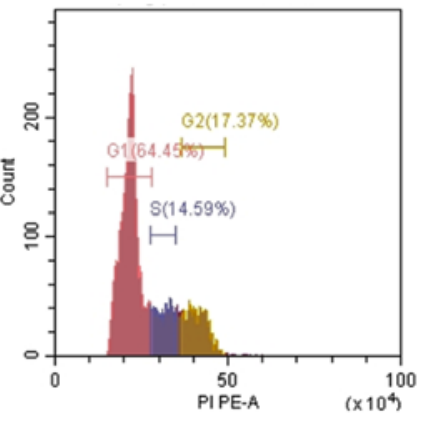

si-ERCC6L-103

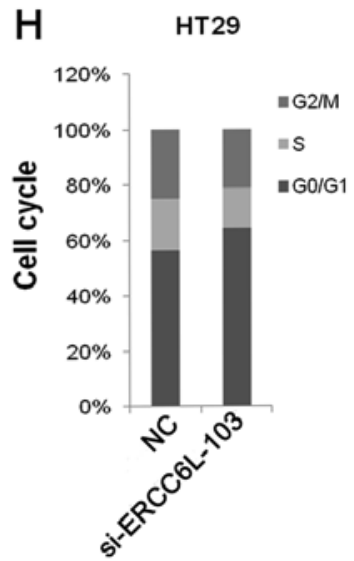

Figure 5. Flow cytometric analysis of apoptosis and the cell cycle in transfected colorectal cancer cells. Representative flow cytometry images showing apoptosis in transfected (A) SW480 and (B) HT29 cells. Apoptosis was measured using Annexin V/PI staining. Representative flow cytometry images for analysis of the cell cycle in (C) SW480 and (D) HT29 cells. Apoptotic cell percentages in the (E) SW480 and (F) HT29 cells, and calculated cell cycle distributions in the (G) SW480 and (H) HT29 cells. The graphs summarize data from three independent experiments. PI, propidium iodide; FITC, fluorescein isothiocyanate; ERCC6L, excision repair cross-complementation group 6 like; NC, negative control; si, small interfering.

colon (36), pancreas (37) and lungs (38). It may be beneficial to investigate the potential association between KRAS and BRAF gene mutations and ERCC6L in different patients with CRC and cell lines, as this may also help to elucidate the underlying mechanism of ERCC6L in tumors.

In conclusion, the present study identified that ERCC6L is upregulated in CRC tissues and cells, and that ERCC6L overexpression is closely associated with tumor size. It also demonstrated that ERCC6L promotes CRC cell growth and proliferation in vitro, possibly via acceleration of the cell cycle. In summary, to the best of our knowledge, the current study provided the first evidence that ERCC6L serves a role in the development of CRC, which indicates that ERCC6L may serve as a novel biomarker and therapeutic target for CRC. Future studies should evaluate the mechanisms of ERCC6L at different stages of the cell cycle and its regulatory signaling pathways.

\section{Acknowledgements}

This abstract was presented at the 8th Shanghai International Conference of Gastroenterology in Shanghai in August 2018, and was published as Abstract ID: 1 in J Dig Dis 19, 696-718, 2018. The abstract was also presented at the 26th United 
European Gastroenterology (UEG) Week, October 20-24, 2018 in Vienna, Austria.

\section{Funding}

The present study was supported by the Applied Basic Research Programs of the Wuhan Science and Technology Department (grant no. 2015061701011642) and the Wuhan University Graduate Student Exchange Program.

\section{Availability of data and materials}

The datasets used and/or analysed during the present study are available fro. the corresponding author on reasonable request

\section{Authors' contributions}

All authors were responsible for the conception and design of the present study. FW and ML were responsible for the provision of the study materials. YX and JY were responsible for the collection and assembly of the data. YX, JY, FW, ML, XQ, YL and JQ performed the data analysis and interpretation. YX, $\mathrm{JY}, \mathrm{XQ}$, and JQ contributed in writing the manuscript. YX, JY, FW, ML, XQ, YL and JQ read and gave the final approval of the manuscript.

\section{Ethical approval and consent to participate}

The study was approved by the Ethics Committee of Zhongnan Hospital of Wuhan University. The patient included in the case provided consent for his or her data to be used in this publication.

\section{Patient consent for publication}

All patients included in this study at the time of data collection provided consent for their data to be used in this publication.

\section{Competing interests}

The authors declare that they have no competing interests.

\section{References}

1. Jemal A, Bray F, Center MM, Ferlay J, Ward E and Forman D: Global cancer statistics. CA Cancer J Clin 61: 69-90, 2011.

2. Siegel R, Miller K and Jemal A: Cancer statistics, 2018. CA Cancer J Clin 68: 7-30, 2018.

3. Chen W, Zheng R, Baade PD, Zhang S, Zeng H, Bray F, Jemal A, Yu XQ and He J: Cancer statistics in China, 2015. CA Cancer J Clin 66: 115-132, 2016.

4. Maurel J and Postigo A: Prognostic and predictive biomarkers in colorectal cancer. From the preclinical setting to clinical practice. Curr Cancer Drug Targets 15: 703-715, 2015.

5. Schreuders EH, Ruco A, Rabeneck L, Schoen RE, Sung JJ, Young GP and Kuipers EJ: Colorectal cancer screening: A global overview of existing programmes. Gut 64: 1637-1649, 2015.

6. Dickinson BT, Kisiel J, Ahlquist DA and Grady WM: Molecular markers for colorectal cancer screening. Gut 64: 1485-1494, 2015

7. Xu Y, Chen X and Li Y: Ercc61, a gene of SNF2 family, may play a role in the teratogenic action of alcohol. Toxicol Lett 157: $233-239,2005$

8. Zhao Z, Zhang G and Li W: Elevated expression of ERCC6 confers resistance to 5-fluorouracil and is associated with poor patient survival in colorectal cancer. DNA Cell Biol 36: 781-786, 2017.
9. Nielsen CF, Huttner D, Bizard AH, Hirano S, Li TN, Palmai-Pallag T, Bjerregaard VA, Liu Y, Nigg EA, Wang LH and Hickson ID: PICH promotes sister chromatid disjunction and co-operates with topoisomerase II in mitosis. Nat Commun 6: $8962,2015$.

10. Baumann C, Körner R, Hofmann K and Nigg EA: PICH, a centromere-associated SNF2 family ATPase, is regulated by Plk1 and required for the spindle checkpoint. Cell 128: 101-114, 2007.

11. Chen XG, Li Y, Zang MX, Pei XR, Xu YJ and Gao LF: cDNA cloning and expression analysis of mouse gene encoding the protein Ercc61 which is a novel member of SNF2 family. Prog Biochem Biophys 31: 443-448, 2004.

12. Santamaria A, Neef R, Eberspächer U, Eis K, Husemann M, Mumberg D, Prechtl S, Schulze V,SiemeisterG, WortmannL, etal: Use of the novel Plk1 inhibitor ZK-thiazolidinone to elucidate functions of Plk1 in early and late stages of mitosis. Mol Biol Cell 18: 4024-4036, 2007.

13. Pu SY, Yu Q, Wu H, Jiang JJ, Chen XQ, He YH and Kong QP: ERCC6L, a DNA helicase, is involved in cell proliferation and associated with survival and progress in breast and kidney cancers. Oncotarget 8: 42116-42124, 2017.

14. Livak KJ and Schmittgen TD: Analysis of relative gene expression data using real-time quantitative PCR and the 2(-Delta Delta C(T)) method. Methods 25: 402-408, 2001.

15. Fearon ER and Vogelstein B: A genetic model for colorectal tumorigenesis. Cell 61: 759-767, 1990.

16. Vogelstein B, Fearon ER, Hamilton SR, Kern SE, Preisinger AC, Leppert M, Nakamura Y, White R, Smits AM and Bos JL: Genetic alterations during colorectal tumor development. N Engl J Med 319: 525-532, 1988.

17. Jagadish N, Parashar D, Gupta N, Agarwal S, Purohit S, Kumar V, Sharma A, Fatima R, Topno AP, Shaha C and Suri A: A-kinase anchor protein 4 (AKAP4) a promising therapeutic target of colorectal cancer. J Exp Clin Cancer Res 34: 142, 2015.

18. Mhaidat NM, Alzoubi KH and Abushbak A: X-box binding protein 1 (XBP-1) enhances colorectal cancer cell invasion. J Chemother 27: 167-173, 2015.

19. Zhou FF, Xie W, Chen SQ, Wang XK, Liu Q, Pan XK, Su F and Feng MH: SLC38A1 promotes proliferation and migration of human colorectal cancer cells. J Huazhong Univ Sci Technolog Med Sci 37: 30-36, 2017.

20. Gil-Raga M, Jantus-Lewintre E, Gallach S, Giner-Bosch V, Frangi-Caregnato A, Safont-Aguilera MJ, Garde-Noguera J, Zorraquino-Pina E, García-Martínez $M$ and Camps-Herrero C: Molecular subtypes in early colorectal cancer associated with clinical features and patient prognosis. Clin Transl Oncol 20: 1422-1429, 2018.

21. Liu J, Deng N, Xu Q, Sun L, Tu H, Wang Z, Xing C and Yuan Y: Polymorphisms of multiple genes involved in NER pathway predict prognosis of gastric cancer. Oncotarget 7: 48130-48142, 2016.

22. Xu Q, Liu JW, He CY, Sun LP, Gong YH, Jing JJ, Xing CZ and Yuan Y: The interaction effects of pri-let-7a-1 rs10739971 with PGC and ERCC6 gene polymorphisms in gastric cancer and atrophic gastritis. PLoS One 9: e89203, 2014.

23. Taghdiri M, Dastsooz H, Fardaei M, Mohammadi S, Farazi Fard MA and Faghihi MA: A novel mutation in ERCC8 gene causing cockayne syndrome. Front Pediatr 5: 169, 2017.

24. Xie H, Li X, Peng J, Chen Q, Gao Z, Song X, Li W, Xiao J, Li C, Zhang T, et al: A complex intragenic rearrangement of ERCC8 in Chinese siblings with Cockayne syndrome. Sci Rep 7: 44271, 2017.

25. He C, Sun M, Wang G, Yang Y, Yao L and Wu Y: Two novel mutations in ERCC6 cause Cockayne syndrome B in a Chinese family. Mol Med Rep 15: 3957-3962, 2017.

26. Truta A, Popon TA, Saraci G, Ghervan L and Pop IV: Novel non invasive diagnostic strategies in bladder cancer. Clujul Med 89: 187-192, 2016.

27. Kurasawa Y and Yu-Lee LY: PICH and cotargeted Plk1 coordinately maintain prometaphase chromosome arm architecture. Mol Biol Cell 21: 1188-1199, 2010.

28. Leng M, Besusso D, Jung SY, Wang Y and Qin J: Targeting Plk1 to chromosome arms and regulating chromosome compaction by the PICH ATPase. Cell Cycle 7: 1480-1489, 2008.

29. Ke Y, Huh JW, Warrington R, Li B, Wu N, Leng M, Zhang J, Ball HL, Li B and Yu H: PICH and BLM limit histone association with anaphase centromeric DNA threads and promote their resolution. EMBO J 30: 3309-3321, 2011.

30. Kaulich M, Cubizolles F and Nigg EA: On the regulation, function, and localization of the DNA-dependent ATPase PICH. Chromosoma 121: 395-408, 2012. 
31. Ma X, Wang L, Huang, Li Y, Yang D, Li T, Li F, Sun L, Wei H, He K, et al: Polo-like kinase 1 coordinates biosynthesis during cell cycle progression by directly activating pentose phosphate pathway. Nat Commun 8: 1506, 2017.

32. Helmke C, Becker S and Strebhardt K: The role of Plk3 in oncogenesis. Oncogene 35: 135-147, 2016.

33. Yen TJ: Polo delivers a PICH to the kinetochore. Cell 128: 20-21, 2007.

34. Baines AT, Xu D and Der CJ: Inhibition of Ras for cancer treatment: The search continues. Future Med Chem 3: 1787-1808, 2011.

35. Sclafani F, Gullo G, Sheahan K and Crown J: BRAF mutations in melanoma and colorectal cancer: A single oncogenic mutation with different tumour phenotypes and clinical implications. Crit Rev Oncol Hematol 87: 55-68, 2013.
36. Zhang J, Zheng J, Yang Y, Lu J, Gao J, Lu T, Sun J, Jiang H, Zhu Y, Zheng Y, et al: Molecular spectrum of KRAS, NRAS, BRAF and PIK3CA mutations in Chinese colorectal cancer patients: analysis of 1,110 cases. Sci Rep 5: 18678, 2015.

37. McAllister F, Bailey JM, Alsina J, Nirschl CJ, Sharma R, Fan H, Rattigan Y, Roeser JC, Lankapalli RH, Zhang H, et al: Oncogenic Kras activates a hematopoietic-to-epithelial IL-17 signaling axis in preinvasive pancreatic neoplasia. Cancer Cell 25: 621-637, 2014.

38. Nguyen-Ngoc T, Bouchaab H, Adjei AA and Peters S: BRAF alterations as therapeutic targets in non-small-cell lung cancer. J Thorac Oncol 10: 1396-1403, 2015.

This work is licensed under a Creative Commons Attribution-NonCommercial-NoDerivatives 4.0 International (CC BY-NC-ND 4.0) License. 